\title{
DENSE BARRELLED SUBSPACES OF UNCOUNTABLE CODIMENSION
}

\author{
STEPHEN A. SAXON AND WENDY J. ROBERTSON
}

(Communicated by William J. Davis)

\begin{abstract}
Let $E$ be a Hausdorff barrelled space. If there exists a dense barrelled subspace $M$ such that $(\operatorname{codim}(M) \geq c) \quad[\operatorname{codim}(M)=\operatorname{dim}(E)]$, we say that ( $M$ is a satisfactory subspace [11]) $[E$ is barrelledly fit], respectively. Robertson, Tweddle and Yeomans [11] proved that $E$ has a barrelled countable enlargement (BCE) if it has a satisfactory subspace. (Trivially) $E$ has a satisfactory subspace if $\operatorname{dim}(E) \geq c$ and $E$ is barrelledly fit. We show that $E$ is barrelledly fit (and $\operatorname{dim}(E) \geq c$ ) if $E \not \neq \varphi$ and either (i) $E$ is an $(L F)$-space, or (ii) $E$ is an infinite-dimensional separable space and the continuum hypothesis holds. Conclusion: barrelledly fit spaces and their permanence properties arise from and advance the study of BCE's.
\end{abstract}

We have shown in [10] that every Hausdorff barrelled space $E$ with $E^{\prime} \neq$ $E^{*}$ has a dense denumerable-codimensional subspace, necessarily barrelled by [13]. In [20] it is shown, assuming a "Condition $\left(2^{\prime}\right)$ " strictly weaker than the continuum hypothesis, that $E$ must always contain a dense uncountablecodimensional subspace. In fact, a number of papers explicitly involve dense infinite-codimensional subspaces of barrelled spaces, including [4, 5, 21] and others individually cited later. This paper focuses on dense large-codimensional subspaces that, in addition, are barrelled, contrasting with the non-barrelled focus of [17].

A Hausdorff topological vector space $E$ is fit [18], [20] if it contains a dense subspace $M$ whose codimension is as large as possible, namely, if $\operatorname{codim}(M)=$ $\operatorname{dim}(E)$. Call $E$ barrelledly fit if $M$ can be chosen so that $M$ is also barrelled. This forces $E$ to be (locally convex and) barrelled; clearly, $E$ is barrelledly fit if and only if there is a dense barrelled subspace with equal dimension and codimension. A barrelledly fit space of dimension $\geq c \quad\left(=2^{\aleph_{0}}\right)$ has a satisfactory subspace; the converse fails (Example 1). Implicit in [3] is a discussion (p. 246) of satisfactory subspaces and a result (Remark (ii)) on barrelledly fit spaces which is generalized here. We improve several parts of [11] and show by later theorems and examples what are the best possible generalizations, in various different senses, of the Abstract's (i). We do not know the answer to

Received by the editors March 21, 1988 and, in revised form, March 20, 1989.

1980 Mathematics Subject Classification (1985 Revision). Primary 46A07; Secondary 46A12. 
the basic question "Is every barrelled, fit space barrelledly fit?". Some further open questions are discussed.

For standard terminology and notation not herein defined or otherwise referenced, one may refer to [6-9]. We assume that all spaces are locally convex and Hausdorff (with real or complex scalar field).

A space $E$ is called a $(d b)$-space if, whenever $E$ is the union of an increasing sequence of subspaces, one of them is dense and barrelled. (See [11] and [14]. Such spaces are also called suprabarrelled in [23].) By an oft-used technique, any infinite-dimensional space $E$ is the union of an increasing sequence of subspaces, each with dimension and codimension equal to the dimension of $E$ (see [13], p. 96, [11], p. 107 and [3], p. 246). This yields immediately:

Proposition 1. Every infinite-dimensional $(d b)$-space is barrelledly fit. (A parallel result: Every infinite-dimensional Baire space is "Baire-ly fit".)

Example 1. A space with a satisfactory subspace need not be barrelledly fit: let $F$ be a space of dimension $c$ with a satisfactory subspace $M$, and let $G$ be a space of dimension $d>c$ with its strongest locally convex topology. Then $E=F \times G$ has a satisfactory subspace $M \times G$. But $E$ is not barrelledly fit. For if $L$ is a dense subspace of $E$ and $\pi$ is the projector of $E$ onto $G$, then $\pi(L)$ is dense in $G$ and so $\pi(L)=G$. Hence $(F \times\{0\})+L=E$, so $\operatorname{codim}(L) \leq \operatorname{dim}(F)=c<d=\operatorname{dim}(E)$, and $E$ is not fit.

Our next Proposition is an immediate consequence of the obvious fact that $E$ (locally convex) is fit if [and only if] $\left(E, \sigma\left(E, E^{\prime}\right)\right)$ is fit, and the fact proved in [18] that if $E$ has a 0 -neighborhood base whose cardinality does not exceed the dimension of $E$, then $E$ is fit [e.g. every infinite-dimensional metrizable space is fit]. We prefer, however, to give a short independent proof: the proposition plays a vital role in one of our main results.

Proposition 2. If $E$ has infinite dimension not exceeded by that of $E^{\prime}$, then $E$ is fit.

Proof. Let $d$ be the (infinite) dimension of $E$, and let $\mathscr{S}$ be a collection of $d$ subspaces each of dimension $d$ such that $E$ is algebraically the direct sum of the members $\left[d^{2}=d\right.$; cf. [10], Example (d)]. For each member $G$ of $\mathscr{S}$, by the Hahn-Banach Theorem

$$
\operatorname{dim}\left(G^{\prime}\right) \leq \operatorname{dim}\left(E^{\prime}\right) \leq d<2^{d}=\operatorname{dim}\left(G^{*}\right)
$$

so $G^{\prime} \neq G^{*}$ and there is a dense 1 -codimensional subspace $G_{0}$ of $G$. It follows that $E_{0}=\operatorname{span}\left(\bigcup_{G \in,}, G_{0}\right)$ is a dense $d$-codimensional subspace of $E$.

Theorem 1. Assume the continuum hypothesis $\left[\aleph_{1}=c\right]$. Let $E$ be a barrelled space containing a subspace $M$ with $\operatorname{dim}(M) \geq c \geq \operatorname{dim}\left(M^{\prime}\right)$. Then $E$ contains a dense barrelled subspace with codimension equal to $\operatorname{dim}(M)$. In particular, $E$ contains a satisfactory subspace.

Proof. Let $N$ be an algebraic complement of $M$ in $E$. 
Case I. $\operatorname{dim}(M)=c$. By Proposition 2, there exists a subspace $M_{0}$ of $M$ which is dense and $c$-codimensional in $M$. Let $B$ be a Hamel basis for an algebraic complement of $M_{0}$ in $M$. There is a collection $\mathscr{B}$ of $2^{c}$ subsets of $B$, each with cardinality $c$, such that $T_{1} \cap T_{2}$ is countable for distinct $T_{1}, T_{2}$ in $\mathscr{B}$, by Theorem 1.3, p. 48 of [8]. The proof proceeds by contradiction: suppose that, for each $T$ in $\mathscr{B}, \quad E_{T}=M_{0}+\operatorname{span}(B \backslash T)+N$ is not barrelled, so that there exists a sequence $S_{T}$ in $E^{\prime}$ which is pointwise bounded on $E_{T}$ but not on $T$; let $R_{T}$ be the sequence of restrictions to $M$. The mapping $T \mapsto R_{T}$ cannot be one-to-one, since there are only $c$ distinct sequences in $M^{\prime}\left[\left(2^{\aleph_{0}}\right)^{\aleph_{0}}=c\right]$. Let $T_{1}, T_{2}$ be distinct with $R_{T_{1}}=R_{T_{2}}$. Then $R_{T_{i}}$ is pointwise bounded on $L=M_{0}+\operatorname{span}\left(B \backslash\left(T_{1} \cap T_{2}\right)\right)$, and so $S_{T_{i}}$ is pointwise bounded on $L+N$, a dense subspace of $E$, barrelled by [13]. Therefore, $S_{T_{i}}$ is $\sigma\left(E^{\prime}, E\right)$-bounded, a contradiction.

Case II. $\operatorname{dim}(M)>c$. For each non- $\sigma\left(M^{\prime}, M\right)$-bounded sequence in $M^{\prime}$, choose one point in $M$ at which the sequence is unbounded. The collection $D$ of all these points has cardinality $\leq c$, so $P=\operatorname{span}(D)$ has codimension in $M$ equal to $\operatorname{dim}(M)[>c]$, and any sequence in $M^{\prime}$ bounded on points of $P$ must be $\sigma\left(M^{\prime}, M\right)$-bounded. Thus the bipolar theorem assures that $P$ is dense in $M$, and $P+N$ is dense in $E$, with codimension equal to $\operatorname{dim}(M)$. Moreover, any sequence in $E^{\prime}$ bounded on points of $P+N$ must be bounded on points of $M+N=E$, so $P+N$ is barrelled.

Corollary 1. Assuming the continuum hypothesis, if $E \not \varphi$ is an infinitedimensional barrelled space with $\operatorname{dim}\left(E^{\prime}\right) \leq c$, then $E$ is barrelledly fit.

Proof. Isomorphically, $\varphi$ is the only $\aleph_{0}$-dimensional barrelled (Hausdorff) space; apply the theorem with $M=E$.

Corollary 2. Assuming the continuum hypothesis, every barrelled space which contains a separable subspace of uncountable dimension has a satisfactory subspace. In particular, every infinite-dimensional separable barrelled space except $\varphi$ (i.e. every separable barrelled space $E$ with $E^{\prime} \neq E^{*}$ ) has a satisfactory subspace; even more, is barrelledly fit.

Proof. The dual of a separable space has dimension $\leq c$.

Assuming the continuum hypothesis, Ian Tweddle in [22] constructed a dense barrelled subspace $\psi$ of $\omega\left[=K^{\aleph_{0}}\right.$, a scalar field product space], demonstrating several interesting properties of $\psi$, including barrelledly fitness. This follows immediately from Corollary 2. One could also observe that, without regard to the continuum hypothesis, if $\aleph_{0}<d \leq c$ then $K^{d}$ is a separable barrelled space. Now assume the continuum hypothesis: the Hahn-Banach Theorem and Corollary 1 assure that every infinite-dimensional barrelled subspace of $K^{d}$ is barrelledly fit, but the latter part of Corollary 2 does not, since $K^{d}$ has nonseparable barrelled subspaces. For example, the subspace $E_{0}$ of members 
of $K^{d}$ having only countably many nonzero coordinates is a dense barrelled nonseparable subspace.

Theorem 5 of [11] states that if $E$ has a satisfactory subspace, then $E$ has a barrelled countable enlargement (BCE). In [19] this result is sharpened by replacing $c$ with $\aleph_{1}$, again assuming "Condition $\left(2^{\prime}\right)$ ". The existence of a BCE is also discussed in [22] and in [1].

Corollary 3. Assuming the continuum hypothesis, every separable infinitedimensional barrelled space except $\varphi$ has a BCE.

Proof. By Corollary 2 and Theorem 5 of [11].

We shall show later (Proposition 6) that every $(L F)$-space except $\varphi$ has a satisfactory subspace, and so a BCE. We may deduce this also here, under the assumption of the continuum hypothesis. For if $E=\underline{\lim } E_{n}$, then one of the Fréchet spaces, say $E_{p}$, is infinite-dimensional $(E \neq \varphi)$. But then $E_{p}$ contains a separable $c$-dimensional subspace $M$, and $M$ remains separable under the coarser topology induced by $E$, and Corollary 2 applies. In fact, in the case of

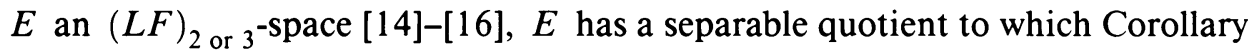
3 or even Proposition 1 applies (see [14], Corollary to Theorem 3 and [15], Theorems 3, 4, 1 and 9), and one is then tempted to try some version of [1], Theorem 5 to see that $E$ has a BCE. A different and fruitful approach develops permanence properties of barrelledly fit spaces, adding to the catalogue of spaces having a satisfactory subspace and so a BCE, and yielding three generalizations of the $(L F)$-space result (Proposition 6, Theorems 3, 4), all without requiring the continuum hypothesis.

Proposition 3. Arbitrary Cartesian products and locally convex direct sums of barrelledly fit spaces are barrelledly fit.

Proof. Obvious.

Example 2. Quotients of barrelledly fit spaces need not be barrelledly fit: let $E \neq\{0\}$ be any barrelledly fit space. Then $E \times \varphi$ is barrelledly fit but has the nonfit $\varphi$ as a quotient. Considering $\{0\} \times \varphi$ shows that: Closed barrelled subspaces of barrelledly fit spaces need not be barrelledly fit. Further, by Example 1: A space may have a barrelledly fit quotient and a nontrivial barrelledly fit closed subspace, without being barrelledly fit.

The next proposition stands in contrast to the last statement. By [12], the three-space problem is true for $P=$ barrelled. (Here we are using the terminology of [2].) With a short straightforward argument, this yields:

Proposition 4. The three-space problem is true for $P=$ barrelledly fit.

Some fit spaces have nonfit countable-codimensional subspaces [18]. However: 
Theorem 2. Every countable-codimensional subspace $M$ of a barrelledly fit space $E$ is barrelledly fit.

Proof. Let $N$ be an algebraic complement of $M$ and let $E_{0}$ be a barrelled dense subspace of $E$ with codimension equal to the dimension of $E$. Set $M_{0}=\left(E_{0}+N\right) \cap M$ and note that, for $E \neq\{0\}, \quad \aleph_{0}<\operatorname{dim}(E)=\operatorname{dim}(E)-$ $\aleph_{0}=\left[\operatorname{codim}\left(E_{0}\right)\right.$ in $\left.E\right]-\aleph_{0} \leq \operatorname{codim}\left(E_{0}+N\right)$ in $E \leq \operatorname{codim}\left(M_{0}\right)$ in $E$, so that, in any case, $\operatorname{codim}\left(M_{0}\right)$ in $E=\operatorname{dim}(E)$. Similarly, $\operatorname{codim}\left(M_{0}\right)$ in $M=\operatorname{codim}\left(M_{0}\right)$ in $E=\operatorname{dim}(E)=\operatorname{dim}(M)$.

Again by the same transfinite arithmetic, if $M_{1}$ is a countable-dimensional subspace of $M, M_{0}+M_{1}$ still has codimension in $M$ equal to the dimension of $M$. Our proof is carried by demonstrating such an $M_{1}$ with $M_{0}+M_{1}$ barrelled and dense in $M$. It is a trivial exercise to show that $M_{0}+N$ contains the dense barrelled space $E_{0}$ and thus is itself barrelled. By [13], $M_{0}$ is barrelled. Again, $F=\bar{M}_{0}+N$ is barrelled, so that any (necessarily countable-dimensional) algebraic complement of $\bar{M}_{0}$ in $F$ is a topological complement and has its strongest locally convex topology by $[13$, p. 92]. $F$, then, is the topological direct sum of two subspaces of $E$, one closed and one complete, so $F$ is closed. Hence $F\left(\supset E_{0}\right)$ is all of $E$. Therefore $\bar{M}_{0}$ is countable-codimensional in $E$, and $\bar{M}_{0} \cap M$ is countable-codimensional in $M$. But $M$ is barrelled, and, again, any algebraic complement $M_{1}$ of $\bar{M}_{0} \cap M$ in $M$ has its strongest locally convex topology, with $M=\left(\bar{M}_{0} \cap M\right) \oplus M_{1}$ topologically. Thus the subspace $M_{0}+M_{1}$ is $M_{0} \oplus M_{1}$, the topological direct sum of two barrelled spaces: $M_{0}+M_{1}$ is barrelled and clearly dense in $M$, completing the proof.

Note. The proof also shows: if $E$ has a satisfactory subspace, so does $M$ (cf. [1], Theorem 6).

In discussing inductive limits, we need the following three simple Lemmas.

Lemma 1. Let $\left(E_{1}, \mathscr{T}_{1}\right)$ and $(E, \mathscr{T})$ be two barrelled spaces, with the first continuously ircluded in the second. If $V$ is an absolutely convex closed subset of $(E, \mathscr{T})$ which absorbs points of a dense barrelled subspace $L$ of $\left(E_{1}, \mathscr{T}_{1}\right)$, then $V$ absorbs points of $E_{1}$. Indeed, $V \cap E_{1}$ is a $\mathscr{T}_{1}$-neighborhood of 0 .

Lemma 2. With $\left(E_{1}, \mathscr{T}_{1}\right)$ and $(E, \mathscr{T})$ as above, if $M$ is a subspace of $E$ such that $M \cap E_{1}$ is a dense barrelled subspace of $\left(E_{1}, \mathscr{T}_{1}\right)$ and such that $E_{1}+M$ is a dense barrelled subspace of $(E, \mathscr{T})$, then $M$ is a dense barrelled subspace of $(E, \mathscr{T})$.

Proof. Let $V$ be any absolutely convex closed subset of $(E, \mathscr{T})$ which absorbs points of $M$. Then by Lemma 1 with $L=M \cap E_{1}, V$ absorbs points of $E_{1}$, and hence points of $E_{1}+M$, and thus points of $E$, now applying Lemma 1 with the two spaces coincident. Therefore $V$ is a $\mathscr{T}$-neighborhood of 0 . It follows that $M$ is dense and barrelled in $(E, \mathscr{T})$.

Lemma 3. Let $(E, \mathscr{T})$ be the (Hausdorff) inductive limit of an increasing system $\left\{\left(E_{\alpha}, \mathscr{T}_{\alpha}\right)\right\}_{\alpha \in \Gamma}$ of barrelled spaces. That is, $\Gamma$ is a totally ordered set; each $E_{\alpha}$ 
is barrelled and for $\alpha, \beta \in \Gamma$ with $\alpha<\beta, E_{\alpha} \subset E_{\beta}$ and $\mathscr{T}_{\beta}$ induces a topology on $E_{\alpha}$ coarser than $\mathscr{T}_{\alpha} ; E=\bigcup_{\alpha \in \Gamma \Gamma} E_{\alpha} ;$ and $\mathscr{T}$ is a Hausdorff locally convex topology, the finest one which induces on $E_{\alpha}$ a topology coarser than $\mathscr{T}_{\alpha}$, for each $\alpha$. If $M$ is a subspace of $E$ such that, for all $\alpha$ in $\Gamma, M \cap E_{\alpha}$ is a dense barrelled subspace of $\left(E_{\alpha}, \mathscr{T}_{\alpha}\right)$, then $M$ is a dense barrelled subspace of $(E, \mathscr{T})$.

Proof. Let $V$ be any absolutely convex closed subset of $(E, \mathscr{T})$ which absorbs points of $M$. Then, for each $\alpha, V \cap E_{\alpha}$ is a $\mathscr{T}_{\alpha}$-neighborhood of 0 by Lemma 1, so $V$ is a $\mathscr{T}$-neighborhood of 0 .

The remaining propositions and theorems generalize Note 3.2, its Corcllary and Corollary 3 to Lemma 3 in [11].

Proposition 5. Let $\left(E_{1}, \mathscr{T}_{1}\right)$ be barrelled and continuously included in the barrelled space $(E, \mathscr{T})$. If $E_{0}$ is a dense barrelled subspace of $\left(E_{1}, \mathscr{T}_{1}\right)$ and $N$ is any algebraic complement of $E_{1}$ in $E$, then $E_{0}+N$ is a dense barrelled subspace of $(E, \mathscr{T})$. In particular, if $\left(E_{1}, \mathscr{T}_{1}\right)$ has a satisfactory subspace, so does $E$.

Proof. Let $M=E_{0}+N$. Then $E=E_{1}+N=E_{1}+M$ and $E_{0}=M \cap E_{1}$, so Lemma 2 applies. Also, the codimension of $E_{0}$ in $E_{1}$ equals that of $E_{0}+N$ in $E$.

Proposition 6. Let $(E, \mathscr{T})$ be the (Hausdorff) inductive limit of an increasing system $\left\{\left(E_{\alpha}, \mathscr{T}_{\alpha}\right)\right\}_{\alpha \in \Gamma}$ of barrelled spaces. If some $\left(E_{\alpha}, \mathscr{T}_{\alpha}\right)$ has a satisfactory subspace, then so does $E$. In particular, every $(L F)$-space except $\varphi$ has a satisfactory subspace and a $\mathrm{BCE}$.

Proof. In general, Proposition 5 applies. In particular, any $(L F)$-space nonisomorphic to $\varphi$ continuously includes an infinite-dimensional Fréchet space $E_{1}$. Thus $\operatorname{dim}\left(E_{1}\right) \geq c$ and $E_{1}$ has a satisfactory subspace by Proposition 1.

Theorem 3. The (Hausdorff) inductive limit $(E, \mathscr{T})$ of an increasing sequence $\left\{\left(E_{n}, \mathscr{T}_{n}\right)\right\}_{n=1}^{\infty}$ of barrelledly fit spaces is barrelledly fit.

Proof. Let $E_{n, d}$ be a dense barrelled subspace of $\left(E_{n}, \mathscr{T}_{n}\right)$ whose dimension and codimension in $E_{n}$ are equal $(n=1,2, \ldots)$. Set $F_{1}=E_{1, d}=M_{1}$ and let $H_{1}=G_{1}$ be any algebraic complement of $F_{1}$ in $E_{1}$. Then $\operatorname{dim}\left(F_{1}\right)=$ $\operatorname{dim}\left(H_{1}\right)=\operatorname{dim}\left(E_{1}\right)$. Let $M_{2}$ be any algebraic complement of $E_{1} \cap E_{2, d}$ in $E_{2, d}$. Then $E_{1}+M_{2} \supset E_{2, d}$. Let $G_{2}$ be any algebraic complement of $E_{1}+M_{2}$ in $E_{2}$, and set

$$
F_{2}=F_{1}+M_{2}, \quad H_{2}=G_{1}+G_{2}=H_{1}+G_{2}
$$

so that $F_{2}$ and $H_{2}$ are algebraically complementary in $E_{2}$ and $F_{1} \subset F_{2}, H_{1} \subset$ $H_{2}$. If $\operatorname{dim}\left(E_{2}\right)=\operatorname{dim}\left(E_{1}\right)$, we clearly have $\operatorname{dim}\left(F_{2}\right)=\operatorname{dim}\left(H_{2}\right)=\operatorname{dim}\left(E_{2}\right)$, and this result still holds by elementary transfinite arithmetic in case $\operatorname{dim}\left(E_{2}\right)>$ $\operatorname{dim}\left(E_{1}\right)$. By Lemma 2, $F_{2}$ is a dense barrelled subspace of $\left(E_{2}, \mathscr{T}_{2}\right)$. Using 
induction, we can continue the process to obtain increasing sequences $\left\{F_{n}\right\}_{n=1}^{\infty}$, $\left\{H_{n}\right\}_{n=1}^{\infty}$ such that $F_{n}$ is a dense barrelled subspace of $\left(E_{n}, \mathscr{T}_{n}\right)$ and $H_{n}$ is an algebraic complement with $\operatorname{dim}\left(F_{n}\right)=\operatorname{dim}\left(H_{n}\right)=\operatorname{dim}\left(E_{n}\right) \quad(n=1,2, \ldots)$. Set

$$
F=\bigcup_{n=1}^{\infty} F_{n}, \quad H=\bigcup_{n=1}^{\infty} H_{n} .
$$

It is clear that $F$ and $H$ are algebraically complementary in $E, \operatorname{dim}(F)=$ $\operatorname{dim}(H)=\operatorname{dim}(E)$, and $F$ is a dense barrelled subspace by Lemma 3 .

Example 3. There are strict inductive limits of (uncountable) increasing systems of barrelledly fit spaces that are not barrelledly fit: Let $E \neq\{0\}$ be any barrelledly fit space, let $\mu$ be the first ordinal whose cardinality exceeds the dimension of $E$, and let $F$ be a vector space with Hamel basis $\left\{x_{\alpha}\right\}_{\alpha \in \mu}$ indexed by $\mu$. For each $\alpha \in \mu$, set $F_{\alpha}=\operatorname{span}\left(\left\{x_{\beta}\right\}_{\beta \leq \alpha}\right)$, given its strongest locally convex topology, and set $G_{\alpha}=E \times F_{\alpha}$. Then $\left\{\bar{G}_{\alpha}\right\}_{\alpha \in \mu}$ is an increasing system of barrelledly fit spaces $\left[\operatorname{dim}\left(F_{\alpha}\right) \leq \operatorname{dim}(E)\right]$ whose inductive limit is strict and is equal to $E \times F$, where $F$ is given its strongest locally convex topology. By the argument of Example $1, E \times F$ is not fit.

Analogues to some of these results, including Theorem 3, Example 3, and the following Theorem 4, are found in [18], with "fit" replacing "barrelledly fit".

Theorem 4. The (Hausdorff) inductive limit of an arbitrary increasing system of infinite-dimensional ( $d b)$-spaces is barrelledly fit.

Proof. Let $(E, \mathscr{T})$ and $\left\{\left(E_{\alpha}, \mathscr{T}_{\alpha}\right)\right\}_{\alpha \in \Gamma}$ be as in Lemma 3 with each $\left(E_{\alpha}, \mathscr{T}_{\alpha}\right)$ an infinite-dimensional $(d b)$-space. We assume that $\Gamma$ has no countable cofinal subset, since the countable case is covered by Theorem 3 . Let $\mathscr{G}$ be the collection of all quadruples $(S, F, H, g)$ such that: $\varnothing \neq S \subset \Gamma ; F$ is a dense barrelled subspace of $L_{S}$, the inductive limit of the increasing system $\left\{\left(E_{\alpha}, \mathscr{T}_{\alpha}\right)\right\}_{\gamma \in S}$, with algebraic complement $H$ (in $\left.L_{S}\right)$; and $g$ is a one-to-one linear map from $F$ onto $H$. By Proposition $1, \mathscr{G}$ is nonempty. We partially order $\mathscr{G}$ by writing $\left(S_{1}, F_{1}, H_{1}, g_{1}\right) \leq\left(S_{2}, F_{2}, H_{2}, g_{2}\right)$ if (and only if) $S_{1} \subset S_{2}$, $F_{1} \subset F_{2}, H_{1} \subset H_{2}$, and $g_{1}=\left.g_{2}\right|_{F_{1}}$. Let $\mathscr{C}$ be a chain in $\mathscr{G}$. Then applying Lemma 3 to the inductive limit of all the spaces $L_{S}$ with $(S, F, H, g) \in \mathscr{C}$, it follows that $\mathscr{C}$ has a least upper bound. By Zorn's Lemma, there is a maximal element $\left(S_{0}, F_{0}, H_{0}, g_{0}\right)$ in $\mathscr{G}$. Now suppose there is some $\alpha$ in $\Gamma$ such that $L_{S_{0}}=F_{0}+H_{0}$ is an infinite-codimensional subspace of $E_{\alpha}$. Let $B$ be a basis of an algebraic complement of $L_{S_{0}}$ in $E_{\alpha}$ and partition $B$ into denumerably many subsets each having the same cardinality as $B$. Applying the technique used in Proposition 1 to the $(d b)$-space $E_{\alpha}=L_{S_{0}}+\operatorname{span}(B)$ yields algebraically complementary subspaces $M$ and $N$ in $\operatorname{span}(B)$ of equal dimension such that $L_{S_{0}}+M$ is dense and barrelled in $\left(E_{\alpha}, \mathscr{T}_{\alpha}\right)$. By equality of dimension, there is a one-to-one linear extension $g_{1}$ of $g_{0}$ mapping $F_{0}+M$ onto $H_{0}+N$. Moreover, $F_{0}+M$ is a dense barrelled subspace of $\left(E_{\alpha}, \mathscr{T}_{\alpha}\right)$ 
by Lemma 2, and the inductive limit space $L_{S_{0} \cup\{\alpha\}}$ is just $\left(E_{\alpha}, \mathscr{T}_{\alpha}\right)$, so that $\left(S_{0} \cup\{\alpha\}, F_{0}+M, H_{0}+N, g_{1}\right)$ is in $\mathscr{G}$, contradicting maximality. Thus it is false to suppose that $L_{S_{0}}$ is infinite-codimensional in any $E_{\alpha}$. It now follows that $L_{S_{0}}$ must be finite-codimensional in $E$ itself: otherwise there would be a strictly increasing sequence $\left\{\alpha_{n}\right\}_{n=1}^{\infty} \subset \Gamma$ such that $L_{S_{0}} \subset E_{\alpha_{1}} \subset E_{\alpha_{2}} \subset \ldots$, with each subspace of (finite) positive codimension in the succeeding one. But as $\left\{\alpha_{n}\right\}_{n=1}^{\infty}$ is not cofinal, there is some $\alpha$ in $\Gamma$ which exceeds each $\alpha_{n}$, thus forcing the contradiction that $L_{S_{0}}$ is infinite-codimensional in $E_{\alpha}$. Let $N$ be a (finite-dimensional) algebraic complement of $L_{S_{0}}$ in $E$. By Proposition 5, $F_{0}+N$ is a dense barrelled subspace of $E$, and clearly, $\operatorname{codim}\left(F_{0}+N\right)$ in $E=\operatorname{dim}\left(H_{0}\right)=\operatorname{dim}\left(F_{0}\right)=\operatorname{dim}\left(F_{0}+N\right)$.

Example 4. There are strict inductive limits of directed systems [7] of infinitedimensional $(d b)$-spaces that are not barrelledly fit: specialize Example 1 by taking $F$ to be a $(d b)$-space. Then $E=F \times G$ is the strict inductive limit of the (by inclusion) directed system $\{F \times N: N$ is a finite-dimensional subspace of $G\}$ of infinite-dimensional $(d b)$-spaces which, as was shown, fails to be fit.

Open questions. In this paper we have answered affirmatively in special cases only the basic, still open question "Does every barrelled space $E$ with $E^{\prime} \neq E^{*}$ have a BCE?". Assuming the generalized continuum hypothesis, [20] shows that $E$ (as above) always contains a barrelled, fit subspace of dimension $\geq c$; thus if every barrelled, fit space is barrelledly fit, then $E$ has a satisfactory subspace and a BCE. In [11] the substitute question was (is) "Does every $E$ (as above) have a satisfactory subspace?"; in this paper, "If $E$ is barrelled and fit, is $E$ barrelledly fit?". All three of the questions seem intrinsically interesting, and as suggested by the reasoning above and in [11], "Yes" to either one of the latter two questions would imply "Yes" to the preceding one(s).

\section{ACKNOWLEDGMENTS}

The first-named author wishes to thank Professor Jean Larson for a stimulating discussion related to Theorem 1.3 of [8].

The originally submitted Corollary 1 contained an error in its proof which was kindly pointed out by Professor Tweddle, to whom the authors are deeply grateful. The first-named author has resolved the difficulty by assuming a condition weaker than the generalized continuum hypothesis and stronger than the singular cardinals hypothesis in his paper "Barrelled spaces $E$ with $\left|E^{\prime}\right| \leq \operatorname{dim}(E)$ " generalizing Theorem 1.

\section{REFERENCES}

1. J. Bonet and Perez Carreras, Remarks on the stability of barrelled-type topologies, Bull. Soc. Roy. Sci. Liège 52 (1983), 313-318.

2. __ On the three-space problem for certain classes of Baire-like spaces, Bull. Soc. Roy. Sci. Liège 51 (1982), 381-385. 
3. M. De Wilde and B. Tsirulnikov, Barrelledness and the supremum of two locally convex topologies. Math. Ann. 246 (1980), 241-248.

4. __ Barrelled spaces with a B-complete completion, Manuscripta Math. 33 (1981), 411-427.

5. L. Drewnowski, A solution to a problem of De Wilde and Tsirulnikov, Manuscripta Math. 37 (1982), 61-64.

6. J. Horváth, Topological vector spaces and distributions, Vol. I, Addison-Wesley, Reading, Massachusetts, 1966.

7. G. Köthe, Topological vector spaces I, Springer-Verlag, Berlin, 1969.

8. K. Kunen, Set theory, North-Holland, Amsterdam, 1980.

9. A. P. Robertson and W. J. Robertson, Topological vector spaces, Cambridge University Press, Cambridge, England, 1964.

10. W. J. Robertson, S. A. Saxon, and A. P. Robertson, Barrelled spaces and dense vector subspaces, Bull. Austral. Math. Soc. 37 (1988), 383-388.

11. W. J. Robertson, I. Tweddle, and F. E. Yeomans, On the stability of barrelled topologies, III, Bull. Austral. Math. Soc. 22 (1980), 99-112.

12. W. Roelcke and S. Dierolf, On the three-space problem for topological vector spaces, Collect. Math. XXXII, Fasc. 1 (1981), 13-35.

13. S. Saxon and M. Levin, Every countable-codimensional subspace of a barrelled space is barrelled, Proc. Amer. Math. Soc. 29 (1971), 91-96.

14. S. A. Saxon and P. P. Narayanaswami, Metrizable (LF)-spaces, $($ db $)$-spaces, and the separable quotient problem, Bull. Austral. Math. Soc. 23 (1981), 65-80.

15. _ ( $(L F)$-spaces, quasi-Baire spaces and the strongest locally convex topology, Math. Ann. 274 (1986), 627-641.

16. _. Metrizable [normable] (LF)-spaces and two classical problems in Fréchet [Banach] spaces, Studia Math. XCIII, Fasc. 1 (1989), 1-16.

17. S. Saxon and A. Wilansky, The equivalence of some Banach space problems, Colloq. Math. 37 (1977), 217-226.

18. S. Saxon, The codensity character of topological vector spaces (in preparation).

19. __ Barrelled spaces and two axiomatic conditions (in preparation).

20. __ The fit and flat components of barrelled spaces (in preparation).

21. B. Tsirulnikov, On conservation of barrelledness properties in locally convex spaces, Bull. Soc. Roy. Sci. Liège 49 (1980), 5-25.

22. I. Tweddle, Barrelled spaces whose bounded sets have at most countable dimension, J. London Math. Soc. (2) 29 (1984), 276-282.

23. M. Valdivia, On suprabarrelled spaces, Funct. Anal., Holomorphy and Approximation Theory, Rio de Janeiro 1978, Lecture Notes in Math. 843 (1981), Springer, pp. 572-580.

Mathematics Department, University of Florida, Gainesville, Florida 32611, U.S.A.

Department of Mathematics, The University of Western Australia, Nedlands, Western Australia 6009 